\title{
Escherichia coli Pneumonia in Combination with Fungal Sinusitis and Meningitis in a Tsunami Survivor after the Great East Japan Earthquake
}

\author{
Ryotaro Igusa, ${ }^{1}$ Sodai Narumi, ${ }^{2}$ Koji Murakami, ${ }^{2}$ Yuko Kitawaki, ${ }^{3}$ Toru Tamii, ${ }^{3}$ \\ Masahiro Kato, ${ }^{4}$ Mineshige Sato, ${ }^{4}$ Masahiro Tsuboi ${ }^{5}$ and Kozo Ota ${ }^{3}$ \\ ${ }^{1}$ Department of Respiratory Medicine, Osaki Citizen Hospital, Osaki, Japan \\ ${ }^{2}$ Department of Respiratory Medicine, Tohoku University Hospital, Sendai, Japan \\ ${ }^{3}$ Department of Internal Medicine, Osaki Citizen Hospital, Osaki, Japan \\ ${ }^{4}$ Department of Neurology, Osaki Citizen Hospital, Osaki, Japan \\ ${ }^{5}$ Department of Radiology, Osaki Citizen Hospital, Osaki, Japan
}

Individuals who survive near drowning often suffer from complicated infections, including multi-organ and polymicrobial events. This pattern may be especially pronounced among patients exposed to infectious agents during catastrophic events like that of the Great East Japan Earthquake and the associated tsunami disaster. We report here on a patient who presented with Escherichia coli (E. coli) pneumonia in combination with fungal sinusitis and meningitis. A 73-year-old woman survived the tsunami that engulfed the Sanriku coast. By the time of hospital admission, the patient exhibited high fever, severe cough, and sputum production. Chest X-ray and CT scan showed consolidation in the left upper lobe. Administration of an antibacterial agent improved this pneumonia. However, the patient's consciousness was increasingly impaired. Brain CT showed the low-density lesions and partial high-density spot in the sinus, which suggests the fungal infection. MRI showed the inflammation in the sinus spread into the central nerve system. The examination of the cerebrospinal fluid showed the low glucose level, high mononuclear cell count, and high $\beta$-D glucan level, the findings of which supported the diagnosis of fungal meningitis. Although the patient improved temporarily in response to combination treatment with anti-fungal agents, no further improvement was seen. In conclusion, this patient, who suffered from infections of pneumonia, sinusitis, and meningitis, presented a quite rare clinical progress. We propose that fungal infection should be taken into consideration in individuals who suffered near drowning, a profile expected to be frequent among tsunami survivors.

Keywords: extended-spectrum $\beta$-lactamase-producing Escherichia coli; fungal meningitis; tsunami-related aspiration pneumonia; tsunami-related sinusitis; tsunami victims

Tohoku J. Exp. Med., 2012, 227 (3), 179-184. (C) 2012 Tohoku University Medical Press

Following the Great East Japan Earthquake of 2011, one of the largest tsunamis in recorded history struck the Pacific coast of the Tohoku district of Japan (Shibahara 2011). About fifteen thousand people were killed, and many thousands more were injured. As seen after the December 26, 2004 Indian Ocean earthquake and tsunami, many individuals survived near-drowning only to suffer from subsequent infections (Allworth 2005; Kao et al. 2005; Maegele et al. 2006). Our hospital, located inland from the Pacific coast, was damaged by the earthquake, but escaped the worst damage of the tsunami. Despite limited resources, we accommodated as many patients from the disaster area as possible.

Tsunami-related aspiration pneumonia is common in tsunami victims (Allworth 2005; Chierakul et al. 2005; Ebisawa et al. 2011). This pneumonia, typically the result of polymicrobial infection, takes the form of lobar pneumonia and often is accompanied by lung abscess (Allworth 2005; Kao et al. 2005). For this reason, long-term treatment with broad-spectrum antibiotics is needed for tsunamiassociated aspiration pneumonia. Additionally, sinusitis often is reported in near-drowning patients, and represents a further difficulty in treatment (Maegele et al. 2006; Baba et al. 2011).

Fungal infection has been reported after near drowning (Nakamura et al. 2011). A diagnosis of fungal meningitis is strongly suggested when the patient shows decreased glucose level and increased mononuclear cell count in the

Received April 6, 2012; revision accepted for publication June 5, 2012. doi: 10.1620/tjem.227.179

Correspondence:Ryotaro Igusa, M.D., Ph.D., Department of Respiratory Medicine, Osaki Citizen Hospital, 2-3-10 Furukawa Senjyujimati, Oskai, Miyagi 989-6183, Japan.

e-mail: ryoigusa@h-osaki.jp 
cerebrospinal fluid (CSF), along with the detection of $\beta$-D glucan in CSF. However, because of the difficulty in isolating fungal pathogens, it is also difficult to diagnose and treat fungal meningitis.

We report here on a tsunami survivor who developed severe pneumonia, sinusitis, and meningitis. We consider this patient extremely thought-provoking in the context of infections following catastrophic events.

\section{Clinical findings of the patient}

A 73-year-old woman, who had not been suffering from chronic disease except hypertension, was swept away when the tsunami engulfed the Sanriku coast. The woman aspirated large amounts of water and mud. Following rescue, the woman took refuge in a local shelter. Two days after the tsunami disaster, the woman exhibited a high fever, severe cough, and sputum production, and so was admitted to a nearby hospital. On admission, chest X-ray and chest computed tomography (CT) scan showed consolidation in the left upper lobe (Fig. 1A). Although the woman was treated with several antibacterial drugs, including panipenem/betamipron, clindamycin, and levofloxacin, her fever persisted, and chest X-rays demonstrated that the lung consolidation had not improved. At 21 days after the tsunami, the patient was transferred to our hospital for further diagnosis and treatment of the chest consolidation and high fever.

On admission, this patient was slightly drowsy, but was easily aroused. There were no wounds on her body. Physical findings were as follows: body temperature of $39.2^{\circ} \mathrm{C}$, blood pressure of $124 / 59 \mathrm{mmHg}$, heart rate of 93 beats/min, respiratory rate of 30 breaths/min, and oxygen saturation of $92 \%$ on approximately $24 \%$ oxygen. The patient displayed equal breath sounds, with coarse crackles in the left lung zone. The leukocyte count was $5,440 / \mathrm{mm}^{3}$, the platelet count was $239,000 / \mathrm{mm}^{3}$, hemoglobin value was $9.7 \mathrm{~g} / \mathrm{dl}$, and hematocrit value was $27.7 \%$. The blood chemistry analysis data were also within normal limits, except for an alkaline phosphatase level of 1,263 IU/1, a total protein level of $6.2 \mathrm{~g} / \mathrm{dl}$, an albumin level of $2.1 \mathrm{~g} / \mathrm{dl}$, and a C-reactive protein level of $0.75 \mathrm{mg} / \mathrm{l}$. Serum electrolyte values were as follows: sodium $126 \mathrm{mEq} / 1$, potassium $3.9 \mathrm{mEq} / \mathrm{l}$, chloride $95 \mathrm{mEq} / \mathrm{l}$; hyponatremia was detected. The serum was negative for $\beta$-D glucan.

Chest X-ray and CT scan of the chest confirmed the consolidation in the left upper lobe (Fig. 1B). These images were consistent with those from the referring hospital (Fig. 1A, B). CT scan of the brain disclosed a low-density lesion in the maxillary sinus and sphenoid sinus, and a partial high-density spot in the sphenoid sinus (Fig. 2B). This abnormality was not detected in the referring hospital (Fig. 2A). The appearance of the high-density spot suggested the possibility of fungal infection. Blood culture was negative, but Escherichia coli was isolated from the culturing of sputum and broncho-alveolar lavage (BAL) specimens.

Because of her poorly general conditions, we could not irrigate the sinus. Accordingly, the patient was administered tazobactam/piperacillin. Four days after admission, sputum culture and BAL fluid yielded extended-spectrum $\beta$-lactamase-producing (ESBL) E. coli. The antibacterial drug was changed to meropenem. This treatment alleviated the chest findings as judged by chest X-ray (Fig. 1C).
A
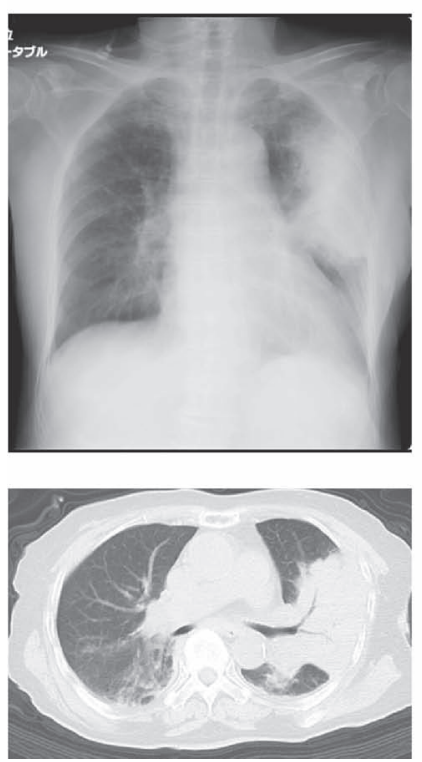

B
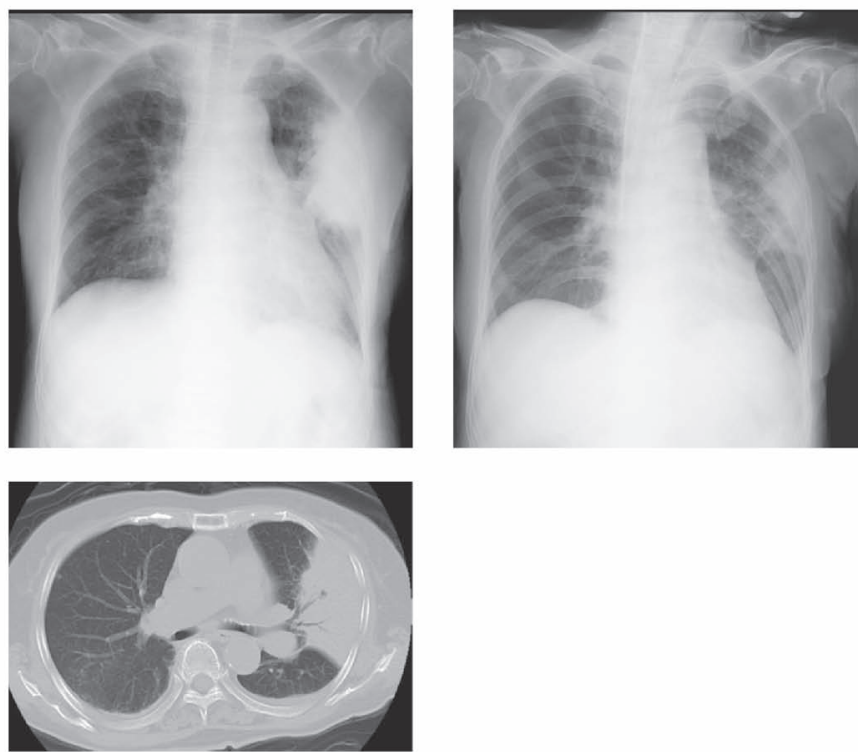

Fig. 1. Chest X-rays and CT scans.

Chest X-rays (upper panels) and CT scans (lower panels) on admission to referring hospital (A), on admission to our hospital (B), and 14 days after admission to our hospital (C). In both (A) and (B), left upper infiltration (X-rays) and air-space consolidation (CT scans) can be seen. Meropenem ameliorated the infiltration (C). 
A

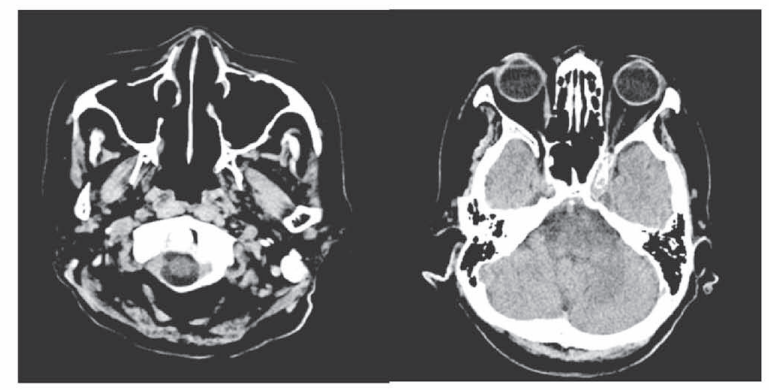

B

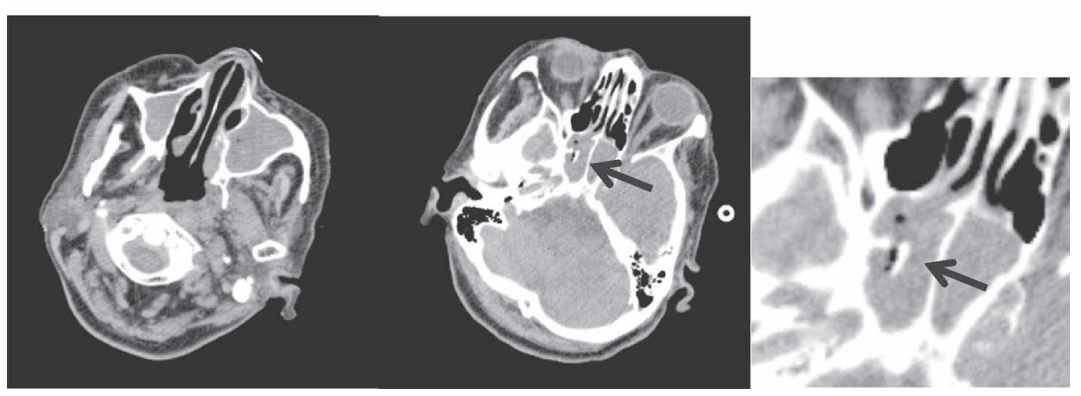

Fig. 2. CT scan of the brain.

CT scans of the brain on admission to the referring hospital (A), and on admission to our hospital (B). In (B), a lowdensity lesion can be seen in the maxillary sinus and sphenoid sinus (left and center). A high-density spot can be seen in the sphenoid sinus (right). Arrows indicate a high-density spot in the sphenoid sinus.

However, the high fever was not improved, and the patient's reduced consciousness worsened, with the woman unable to open her eyes, but able to make brief responses to questions. Hyponatremia persisted, and serum osmotic pressure was lower than uremic osmotic pressure. Anti-diuretic hormone (ADH) was detected at a high level of $4.3 \mathrm{pg} / \mathrm{ml}$. These results were consistent with a diagnosis of syndrome of inappropriate secretion of ADH (SIADH).

In order to diagnose the cause of SIADH and to check the progress of sinusitis, brain MRI was performed. The brain MRI showed that the size of the occupying lesion had decreased in the left maxillary sinus, and was now present not only in the right maxillary and sphenoid sinus, but also in the ethmoid sinus (Fig. 3A, B, C). A contrast-enhanced MRI showed that the high-density lesion had spread out over the cavernous sinus (Fig. 3D). These images strongly suggested the worsening of highly complicated sinusitis, with possible inflammation of the central nervous system (CNS). We therefore performed nasal fiberscope and lumbar puncture. Pus was detected in the right middle nasal meatus and in both the ethmoid and sphenoid sinuses, extending down to the upper pharynx. ESBL $E$. coli was isolated upon culturing of the pus from the nasopharynx.

Examination of the CSF revealed a cell count of 325/ $\mu \mathrm{l}$, and especially a mononuclear cell count of $195 / \mu \mathrm{l}$ (cells are undetectable in normal CSF), glucose level of $33 \mathrm{mg} / \mathrm{dl}$ (normal glucose level in CSF is more than a half of blood glucose level), and a $\beta$-D glucan level of $23.78 \mathrm{pg} / \mathrm{ml}(\beta$-D glucan is undetectable in normal CSF). No pathogens were isolated from the CSF.

These results suggested fungal meningitis rather than bacterial meningitis. Combination treatment with anti-fungals (micafungin sodium and voriconazole) and antibacterials (meropenem and vancomycin) was begun. Although the CSF parameters and level of consciousness improved slightly, the patient's clinical condition worsened on the 12th day after the initiation of combination treatment (Fig. 3). Subsequently, the patient died owing to meningitis.

\section{Discussion}

Tsunami-related aspiration pneumonia is common among tsunami victims (Allworth 2005; Chierakul et al. 2005; Ebisawa et al. 2011). People who survive the wave frequently aspirate not only water, but also solid and particulate matter. Pneumonia after the aspiration of water or after near-drowning is often polymicrobial, with causative agents including aeromonads, pseudomonads, streptococci, E. coli, and others (Kao et al. 2005; Maegele et al. 2006; Ebisawa et al. 2011). In the patient discussed here, polymicrobial infection was supposed, but only $E$. coli was isolated from sputum culture and BAL fluid. We suspect that other infecting organisms already had been inhibited and/or eliminated by one or more of the anti-bacterial agents that had been administered in the referring hospital.

E. coli infectious pneumonia is rarely seen in community-acquired pneumonia (CAP), but is detected in 3\% to $5 \%$ of patients with healthcare-associated pneumonia (HCAP) (Roson et al. 2001). E. coli infection has been 
A

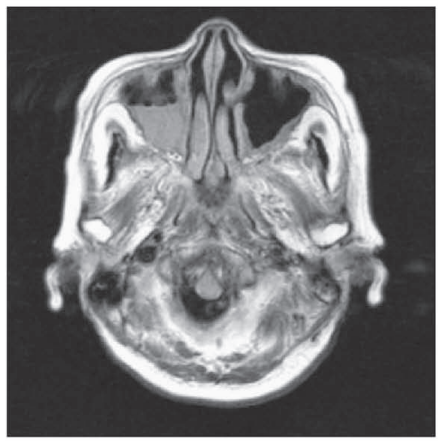

C

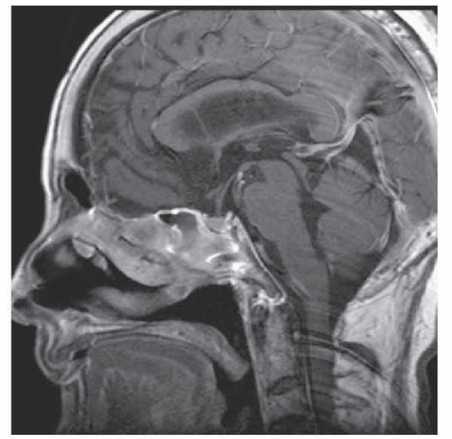

B

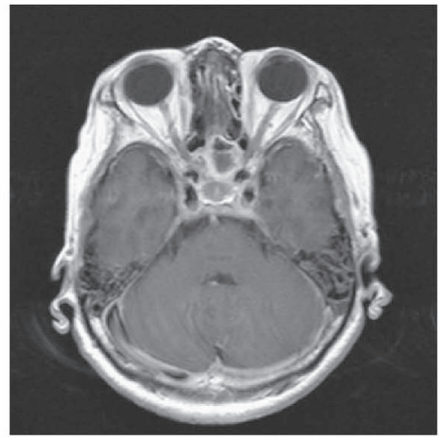

D

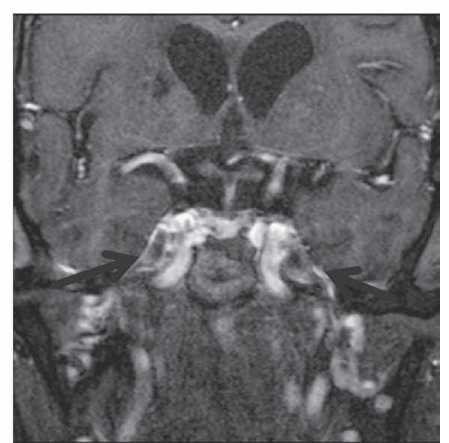

Fig. 3. Contrast-enhanced MRI of the brain.

A contrast-enhanced MRI at 12 days after admission. A low-intensity area with peripheral capsular enhancement can be seen in the left maxillary sinus (A) and the sphenoid sinus and ethmoid sinus (B, C). In (D) high-density lesions (indicated by arrows) can be seen around the cavernous sinus.

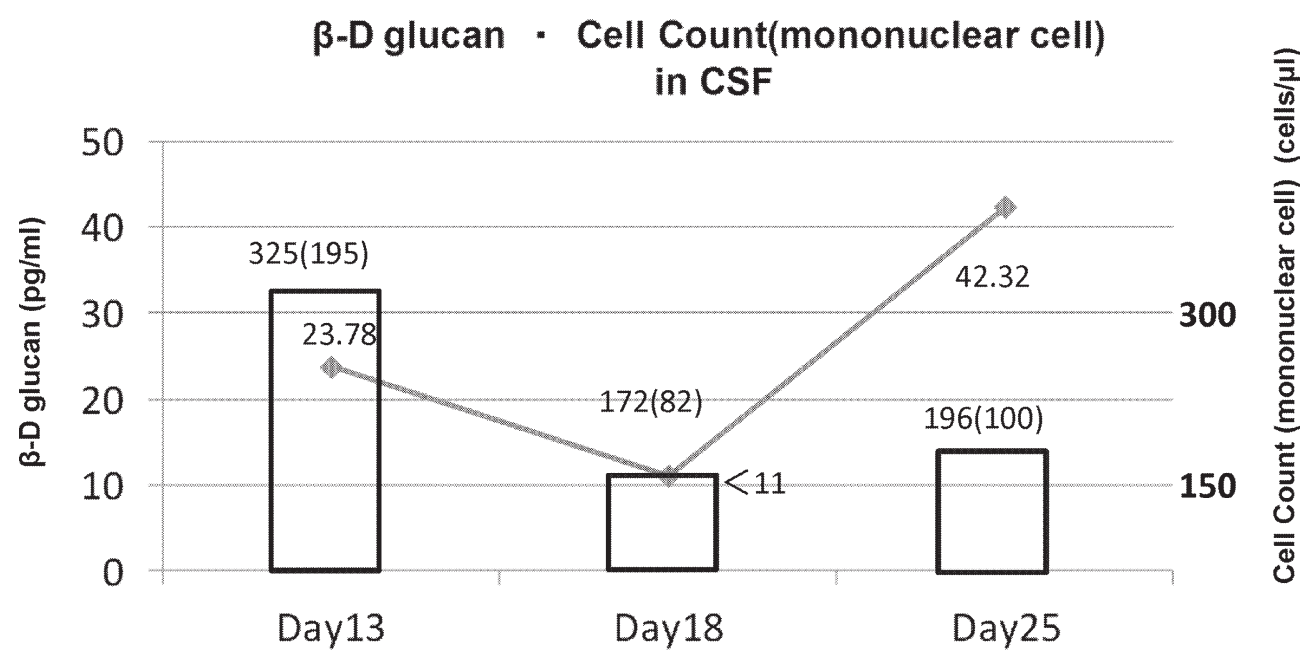

Fig. 4. Clinical course.

This graph plots CSF $\beta$-D glucan levels (in pg/ml; y-axis; left side; line graph) and the cell count (cells/ $\mu$; $y$-axis; right side; bar graph) as a function of the number of days after admission (x-axis).

reported in patients with tsunami-associated aspiration pneumonia, and the isolated organisms often include drugresistant pathogens (Maegele et al. 2006; Ebisawa et al. 2011). In sputum cultures, E. coli may be isolated as a contaminant (Ruiz et al. 2008). The possibility of contamina- tion is higher in debilitated patients, whose oropharynx is frequently colonized by gram-negative bacilli (GNB). The oropharynx of near-drowning patients can also be colonized by GNB. Because ESBL-producing E. coli was isolated from the BAL fluid of the present patient, we administered 
a high dose of a broad-spectrum anti-bacterial drug.

Sinusitis has been reported as a tsunami-associated infection (Maegele et al. 2006; Baba et al. 2011). Water, soil, sand, and particulate matter are thought to enter the sinuses through the ostia because of the high velocity of tsunami waves and then remain in the sinuses of the nearly drowned victims. Polymicrobial and Aspergillus fumigatus infections have been reported (Maegele et al. 2006; Baba et al. 2011). In our patient, ESBL E. coli was isolated. Improvement of the left maxillary sinusitis and of pneumonia followed the use of anti-bacterial drugs. However, sinusitis spread throughout the cavernous sinus. A partial high-density spot in the sphenoid sinus was also observed. These images suggested that other pathogens, such as fungus, were present and not controlled by anti-bacterial agents.

Ethmoid and sphenoid sinuses are anatomically surrounded by the turkish saddle, the superior orbital fissure, the cavernous sinus, and the internal jugular vein. Therefore, severe ethmoid and sphenoid sinusitis are often accompanied by the meningitis, cranial nerve disorder, and the cavernous sinus embolism (Saitoh et al. 2003; Botturi et al. 2006).

Probable pathophysiological mechanisms of the CNS infection include the direct invasion or hematogenous spread. In the patient discussed here, $\beta$-D glucan was not detected in serum, and the leukocyte count and CRP remained within normal ranges. These results proved that this patient did not have bacillemia. Her meningitis was not caused by hematogenous spread, but probably caused by local invasion from sinusitis.

In general, fungal meningitis presents as opportunistic infections in immunosuppressed patients. Fungal meningitis is difficult to diagnose. In this patient, meningitis was strongly suggested by the decreased glucose level and increased mononuclear cell count seen in the patient's CSF. The additional detection of $\beta$-D glucan in the CSF, combined with the negative result for Cryptococcus antigen, suggests a diagnosis of non-cryptococcal (e.g., Aspergillus or Candida) fungal meningitis. The pathogen responsible for the sinusitis and meningitis was not identified from our patient. In tsunami victims, Scedosporium apiospermum infection has been reported (Nakamura et al. 2011). In tsunami waters, various types of fungi, including Scedosporium apiospermum, may be transmitted by the water, soil, and mud carried by the flood. Our patient, who was not an immunocompromised host, might have been infected by one or more of these fungal pathogens.

There are no precise guidelines for treatment of unknown fungal meningitis. Amphotericin B, which is a broad-spectrum, strong anti-fungal drug, has severe toxic side-effects that make the compound difficult to use in debilitated patients. We therefore opted for treatment with micafungin sodium and voriconazole, which are also broadspectrum anti-fungal drugs, but have fewer toxic sideeffects than amphotericin B. Additionally, voriconazole exhibits good CSF permeation (Lutsar et al. 2003). However, we note that Nakamura et al. (2011) reported that Scedosporium apiospermum infection was cured not by voriconazole, but by amphotericin B. If our patient was suffering from a Scedosporium apiospermum infection, the combination treatment that we used would not have been sufficient.

Progression of fungal infection is slower than that of bacterial infection. At two days after the tsunami, X-ray and CT scan revealed pneumonia, but did not detect sinusitis (Fig. 2). Therefore, priority was given to medical treatment of the pneumonia, although (retrospectively) the prescription of an antifungal drug may have been of additional value to this specific patient. The clinical course of this patient demonstrates the importance of the early detection and treatment of latent fungal infections.

\section{Conflict of Interest}

The authors have no conflict of interest to declare in relation to this article.

\section{References}

Allworth, A.M. (2005) Tsunami lung: a necrotising pneumonia in survivors of the Asian tsunami. Med. J. Aust., 182, 364.

Baba, S., Kondo, K., Kanaya, K., Suzukawa, K., Sato, T., Kurata, H., Hiruma, T., Fujita, H., Yahagi, N. \& Yamasoba, T. (2011) Tsunami sinusitis. Lancet, 378, 1116.

Botturi, A., Salmaggi, A., Pollo, B., Lamperti, E., Erbetta, A. \& Boiardi, A. (2006) Meningitis following relapsing painful ophthalmoplegia in aspergillus sphenoidal sinusitis: a case report. Neurol. Sci., 27, 284-287.

Chierakul, W., Winothai, W., Wattanawaitunechai, C., Wuthiekanun, V., Rugtaengan, T., Rattanalertnavee, J., Jitpratoom, P., Chaowagul, W., Singhasivanon, P., \& White, N.J., Day, N.P. \& Peacock, S.J. (2005) Melioidosis in 6 tsunami survivors in southern Thailand. Clin. Infect. Dis., 41, 982-990.

Ebisawa, K., Yamada, N., Okada, S., Suzuki, Y., Satoh, A., Kobayashi, M. \& Morikawa, N. (2011) Combined Legionella and Escherichia coli lung infection after a tsunami disaster. Intern. Med., 50, 2233-2236.

Kao, A.Y., Munandar, R., Ferrara, S.L., Systrom, D.M., Sheridan, R.L., Cash, S.S. \& Ryan, E.T. (2005) Case records of the Massachusetts General Hospital. Case 19-2005. A 17-yearold girl with respiratory distress and hemiparesis after surviving a tsunami. N. Engl. J. Med., 352, 2628-2636.

Lutsar, I., Roffey, S. \& Troke, P. (2003) Voriconazole concentrations in the cerebrospinal fluid and brain tissue of guinea pigs and immunocompromised patients. Clin. Infect. Dis., 37, 728-732.

Maegele, M., Gregor, S., Yuecel, N., Simanski, C., Paffrath, T., Rixen, D., Heiss, M.M., Rudroff, C., Saad, S., Perbix, W., Wappler, F., Harzheim, A., Schwarz, R. \& Bouillon, B. (2006) One year ago not business as usual: wound management, infection and psychoemotional control during tertiary medical care following the 2004 Tsunami disaster in southeast Asia. Crit. Care, 10, R50.

Nakamura, Y., Utsumi, Y., Suzuki, N., Nakajima, Y., Murata, O., Sasaki, N., Nitanai, H., Nagashima, H., Miyamoto, S., Yaegashi, J., Hatakeyama, T., Shibano, Y., Yarita, K., Kamei, K., Nakadate, T., Endo, S., Terayama, Y. \& Yamauchi, K. (2011) Multiple Scedosporium apiospermum abscesses in a woman survivor of a tsunami in northeastern Japan: a case report. J. Med. Case Reports, 5, 526. 
Roson, B., Carratala, J., Dorca, J., Casanova, A., Manresa, F. \& Gudiol, F. (2001) Etiology, reasons for hospitalization, risk classes, and outcomes of community-acquired pneumonia in patients hospitalized on the basis of conventional admission criteria. Clin. Infect. Dis., 33, 158-165.

Ruiz, L.A., Zalacain, R., Gomez, A., Camino, J., Jaca, C. \& Nunez, J.M. (2008) Escherichia coli: an unknown and infrequent cause of community acquired pneumonia. Scand. J. Infect. Dis., 40, 424-427.

Saitoh, A., Beall, B. \& Nizet, V. (2003) Fulminant bacterial meningitis complicating sphenoid sinusitis. Pediatr. Emerg. Care, 19, 415-417.

Shibahara, S. (2011) The 2011 Tohoku earthquake and devastating tsunami. Tohoku J. Exp. Med., 223, 305-307. 